\title{
Diacronie
}

Studi di Storia Contemporanea

$\mathrm{N}^{\circ} 30,2$ | 2017

Ponti fra nazioni e continenti

\section{Renato Agazzi, La rivoluzione del 1848, vol. 1, La nascita della patria}

Edoardo Grassia

\section{Q OpenEdition \\ Journals}

Edizione digitale

URL: http://journals.openedition.org/diacronie/5869

DOI: $10.4000 /$ diacronie.5869

ISSN: 2038-0925

Editore

Association culturelle Diacronie

Notizia bibliografica digitale

Edoardo Grassia, «Renato Agazzi, La rivoluzione del 1848, vol. 1, La nascita della patria », Diacronie

[Online], Nº 30, 2 | 2017, documento 6, Messo online il 29 juillet 2017, consultato il 24 septembre 2020.

URL : http://journals.openedition.org/diacronie/5869; DOI : https://doi.org/10.4000/diacronie.5869 


\title{
Diacronie
}

Studi di Storia Contemporanea

30, 2/2017

Ponti fra nazioni e continenti: diplomazia, immaginari e conoscenze tecniche

\section{RECENSIONE: Renato AGAZZI, La rivoluzione del 1848, vol. 1, La nascita della patria, Udine, Gaspari, 2015, $191 \mathrm{pp}$.}

\author{
A cura di Edoardo GRASSIA
}

Per citare questo articolo:

GRASSIA, Edoardo, «Renato AGAZZI, La rivoluzione del 1848, vol. 1, La nascita della patria, Udine, Gaspari, 2015,191 pp.», Diacronie. Studi di Storia Contemporanea : Ponti fra nazioni e continenti: diplomazia, immaginari e conoscenze tecniche, 30, 2/2017, 29/7/2017,

URL: < http://www.studistorici.com/2017/07/29/grassia_numero_30/ >

Diacronie Studi di Storia Contemporanea $\rightarrow \underline{\text { http://www.diacronie.it }}$ Rivista storica online. Uscita trimestrale.

redazione.diacronie@hotmail.it

Comitato di direzione: Naor Ben-Yehoyada - João Fábio Bertonha - Christopher Denis-Delacour - Maximiliano Fuentes Codera Anders Granås Kjøstvedt - John Paul Newman - Deborah Paci - Niccolò Pianciola - Spyridon Ploumidis - Wilko Graf Von Hardenberg

Comitato di redazione: Jacopo Bassi - Luca Bufarale - Gianluca Canè - Fausto Pietrancosta - Alessandro Salvador - Matteo Tomasoni Diritti: gli articoli di Diacronie. Studi di Storia Contemporanea sono pubblicati sotto licenza Creative Commons 3.0. Possono essere

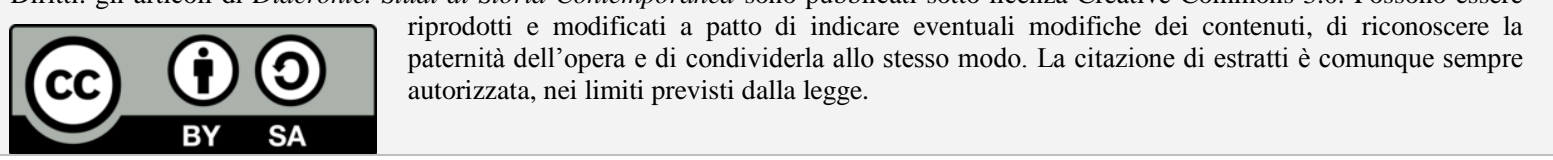




\title{
6/ RECENSIONE: Renato AGAZZI, La rivoluzione del 1848, vol. 1, La nascita della patria, Udine, Gaspari, 2015, 191 pp.
}

\author{
A cura di Edoardo GRASSIA
}

Dal Mito del vampiro in Europa ${ }^{1}$ del 1979 all'analisi del romanzo gotico inglese con I romantici dell'orrido ${ }^{2}$ del 1984, Renato Agazzi si è confrontato, nel 2006, anche con uno studio che differisce profondamente dai sui precedenti per collocarsi nell'ambito della storia militare, presentando Giulio Cesare, stratega in Gallia ${ }^{3}$. Fu probabilmente una esperienza coinvolgente tanto che nel 2015 l'autore - restando nell'alveo della storia militare, ma spostando il proprio focus dalla storia antica al Risorgimento italiano - ha proposto un nuovo studio: La rivoluzione del 1848. La nascita della patria. Volume I: Dall'inizio delle ostilità alla battaglia di Goito. Un testo che, come indicato, è il primo di una trilogia che si completerà con il Da Custoza a Novara e Gli assedi.

Renato Agazzi è dottore in scienze naturali, non propriamente uno storico, ma un grande appassionato di storia militare, materia coltivata anche grazie alla professione di libraio di testi d'antiquariato.

Prima di introdurci in una disamina del testo, vogliamo proporre qualche riflessione sull'argomento. In Italia sono sempre più rari gli studi di storia militare, soprattutto quelli focalizzati sul periodo preunitario. Tale limite non è probabilmente dovuto alla mancanza di interesse per questi studi - comunque un interesse ristretto che trova poco spazio in ambito accademico - ma per alcune difficoltà tra le quali occorre segnalare il fatto che pochi ricercatori si vogliono o possono confrontare con un approccio che deve necessariamente tener conto di componenti umane e tecniche militari, con la scarsa e datata bibliografia e la difficoltosa ricerca archivistica agevolata da pochi istituti, per quanto molto validi.

Pubblicare un testo come quello in esame nel 2015, ovvero nell'anno in cui la maggior parte delle superfici degli scaffali di storia sono occupate da vecchie e nuove ricerche legate alla commemorazione della Grande Guerra che troppo spesso evitano di guardare prima del 1914, non è cosa di poco conto.

\footnotetext{
${ }^{1}$ AGAZZI, Renato, Il mito del vampiro in Europa, Poggibonsi, Lalli, 1979.

${ }^{2}$ ID., I romantici dell'orrido. Uno studio sul romanzo gotico inglese, Poggibonsi, Lalli, 1984.

${ }^{3}$ ID., Giulio Cesare stratega in Gallia, Pavia, Iuculano, 2006.
} 
Infine, un'ultima nostra riflessione è rivolta al fatto che nonostante celebrazioni di anniversari, di dialoghi sulla bandiera tricolore e sull'unità storica e sociale italiana, l'ultimo testo di storia militare legato al Risorgimento italiano, per quanto ancora valido, è comunque il saggio di Pieri datato $1962^{4}$ - fatta eccezione per il testo specifico sulla campagna navale 1860-1861 pubblicato nel $2012^{5}$ e pochissimo altro ${ }^{6}$ - a differenza della memorialistica e della storia sociale del Risorgimento che, dagli anni Ottanta e nei più recenti anni Novanta del Novecento e inizi del Duemila hanno fornito numerose produzioni letterarie ${ }^{7}$.

Agazzi è molto diretto nel fornire delle chiavi di lettura al suo studio. Non vuole dare una visione dal basso. Egli non ha voluto prendere in considerazione anche diaristica e carteggio dei soldati perché giudicate testimonianze «molto interessanti da un punto di vista psicologico e sociale, ma che si possono rintracciare sempre uguali a se stesse, in ogni battaglia ${ }^{8}{ }$. Questa è una scelta che poco ci convince, soprattutto se si vuole focalizzare il testo anche sulla nascita del sentimento patriottico-unitario e non considerare la "patria" solo da un punto di vista di annessione territoriale derivante da una conquista militare 9 . Un'analisi di questa documentazione - scartata a priori anche perché valutata «di una monotona ripetitività» e non così decisiva da "cambiare il quadro d'insieme di una battaglia» ${ }^{10}$ - avrebbe invece potuto porre in luce nuove informazioni sulla vita nei campi di battaglia, sul rapporto tra le diverse forze che, per coscrizione o volontariamente, si batterono contro gli austriaci - o contro gli italiani, guardando dall'opposta direzione - e sull'eventuale reciproca conoscenza dei soldati provenienti dalle diverse regioni d'Italia, tra i punti evidentemente fondamentali della "nascita della patria", di cui al titolo del libro. Sarebbe stato interessante ricercare su queste fonti dirette anche notizie più specificatamente tecnico-militari.

Un importante merito dell'opera, dichiarato dall'autore, è quello di dar voce ad entrambi gli schieramenti in lotta. Le guerre di indipendenza costituiscono sicuramente un importante retaggio nazionale, ma oggi, in termini di storia europea - cui, a nostro avviso, anche la storia militare deve necessariamente tendere - appare indispensabile studiare lo stesso evento dalle diverse postazioni che, in questo specifico caso, sono tanto sabaude quanto austriache, ma anche

\footnotetext{
${ }^{4}$ PIERI, Piero, Storia militare del Risorgimento. Guerre e insurrezioni, Torino, Einaudi, 1962.

${ }^{5}$ BATTAGLIA, Antonello, Il Risorgimento sul mare. La campagna navale 1860-1861, Roma, Edizioni Nuova Cultura, 2012.

${ }^{6}$ Tra i più recenti, si segnala SCARDIGLI, Marco, Le grandi battaglie del Risorgimento, Milano, BUR, 2011.

${ }^{7}$ Si segnalano, tra gli altri, BANTI, Alberto Mario, Il Risorgimento Italiano, Roma-Bari, Laterza, 2009; CAVOUR, Camillo Benso, Discorsi per Roma capitale, Roma, Donzelli, 2010; PÉCOUT, Gilles, Il lungo Risorgimento. La nascita dell'Italia contemporanea, Milano, Mondadori, 1999; RIALL, Lucy, Il Risorgimento. Storia e interpretazioni, Roma, Donzelli, 1997; ALBERTINI, Mario, Il Risorgimento e l'unità europea, Napoli, Guida, 1979; Giuseppe Cesare Abba e la memorialistica garibaldina, Brescia, Ateneo di Brescia, 1980.

${ }^{8}$ AGAZZI, Renato, La rivoluzione del 1848. La nascita della patria, Udine, Gaspari, 2015, p. 10.

9 Il termine-concetto "patria", oltre al "territorio", include anche il "popolo" che vi risiede e che per nascita, lingua, cultura, storia e tradizioni vi si sente di appartenere.

${ }^{10}$ AGAZZI, Renato, La rivoluzione del 1848. La nascita della patria, cit., p. 10.
} 
toscane o papaline o, nel complesso, meridionali. Renato Agazzi ci propone proprio questo, risultando tra i pochi autori che permette quindi una lettura comparativa degli eserciti in lotta e dei rispettivi comandanti.

Il 18 marzo 1848 Milano insorse e l'autore rivolge da subito il suo sguardo su ciò che accadde non solo nel vicino Piemonte ma in tutti i diversi stati italiani. Carlo Alberto viene analizzato, almeno inizialmente, sotto un profilo più psicologico che militare confrontando il suo operato con la dimensione repubblicana della rivolta di Milano. Nella città lombarda, infatti, erano i mazziniani repubblicani e i democratici riformisti la componente fortemente maggioritaria che guidò l'insurrezione. L'espansione della rivolta negli stati vicini, o l'esempio da questa fornito, avrebbe potuto coincidere con la diffusione di un pensiero politico ostile e antitetico alle monarchie mettendo così a forte rischio le vicine case regnanti. La volontà di arrestare la rivolta scoppiata a Milano e di impedire nuove insurrezioni, da questo punto di vista, poteva allora divenire un collante tra le corona piemontese e quella austriaca, avendo entrambe la comune necessità di combattere il pensiero diffuso dai seguaci del Mazzini o del Cattaneo. E questa analisi viene ripetuta e proposta da Agazzi anche per gli altri regnanti, il Duca di Toscana, Papa Pio IX e Ferdinando II di Borbone. Per questi, l'autore fornisce anche delle opportune valutazioni relative al fatto che, oltre alla necessità di sopprimere sentimenti antimonarchici, dovevano parimenti tener conto di altri fattori, spesso contrastanti, come l'autorizzazione all'arruolamento di truppe volontarie che chiedevano di partire per il Lombardo-Veneto o l'opportunità di inviare proprie truppe regolari, la volontà o meno di rompere i rapporti con l'Austria, la gestione dei movimenti di ribellione interni ai propri territori - soprattutto nel meridione borbonico - e le mire espansionistiche di Carlo Alberto su un futuro assetto unitario dell'Italia sotto la corona sabauda. Affrontare anche questi argomenti, pur senza giustamente entrare troppo in profondità, ha permesso all'autore di trasmettere anche il senso di disagio sociale presente in molte parti della penisola. Esponendo questa storia dall'alto Agazzi, prima di immergersi nei campi di battaglia, raccoglie e riassume questi pensieri - traendoli dalle memorie di uomini contemporanei ai fatti o dalla datata storiografia della seconda metà dell'Ottocento indicata nelle note - in modo tale da poter fornire al lettore una giusta panoramica e degli appropriati strumenti che serviranno, successivamente, per spiegare anche le dinamiche militari o, quantomeno, le decisioni e gli ordini impartiti dai regnanti e dagli alti comandi.

I soggetti principali del testo sono, quindi, gli stessi regnanti e i loro generali. Per ciascuno Agazzi redige una breve biografia che, per quanto possa rendere più pesante la lettura, riesce a far comprendere al lettore più esigente le scelte che ciascun singolo operò concretamente quando dovette guidare i propri uomini in battaglia. Le pagine dedicate a Carlo Alberto e Radetzky sono ben concentrate e in grado di suscitare curiosità nel lettore. 
Come detto, una storia dall'alto, ma non mancano alcune pagine descrittive degli eserciti coinvolti e delle diverse battaglie che, per la terminologia usata, sono di profonda "cultura militare" e fanno trasparire una certa precisione nello studio, seppur probabilmente risulteranno di non facile lettura per i non avvezzi.

Agazzi scrive brevemente anche di alcuni riferimenti simbolici, soprattutto sull'uso del tricolore e sul fatto che ad esso fu affiancato talvolta lo scudo sabaudo. Sarebbe stato interessante aprire un discorso più ampio di tali episodi, non solo per "la nascita della patria", ma, volendo concentrarsi sulla specifica storia militare, anche per l'importanza che questi assunsero nei campi di battaglia.

Le battaglie. Pastrengo (30 aprile 1848), Santa Lucia (6 maggio 1848), Vicenza (24 maggio 1848), gli assedi di Peschiera, la battaglia di Curtatone e Mantova e, infine, quella di Goito del 30 maggio 1848 descrivono anche altri avvenimenti militari che si svolsero nello stesso periodo, come la rivolta siciliana e quella calabrese. Questi eventi, su cui non ci soffermeremo singolarmente, vengono ricostruiti dall'autore con accurata precisione, riportando per ciascuno di essi più cartine geografiche di grande ausilio non solo per focalizzare i campi di battaglia ma anche per seguire bene i singoli avvenimenti e il loro procedere, l'organizzazione delle forze in campo, gli ordini impartiti e le relative decisioni di volta in volta prese, facendo spesso riferimento alle relazioni e ai resoconti scritti dai principali comandanti, editi ormai all'inizio del Novecento e difficilmente reperibili ${ }^{11}$. L'autore, oltre a trarne indicazioni, utilizza spesso direttamente la fonte e, talvolta, ne propone attente analisi relative al giusto impiego delle truppe, agli errori di valutazione, come nel caso degli assedi di Peschiera, Mantova e Verona, e i relativi insegnamenti che ne poté trarre Carlo Alberto. Come accennato, un elemento di sicuro interesse è l'osservare gli avvenimenti da entrambi gli schieramenti in guerra: proprio a partire dall'analisi delle relazioni e dei rapporti, Agazzi, come scrive chiaramente nelle indicazione metodologiche riportate in prefazione $^{12}$, si impegna a non dare solo le informazioni che è possibile trarre dagli scritti dei comandanti in campo "italiani” ma, per una più completa analisi, concede, per la trattazione delle medesime vicende, analoghi spazi anche ai resoconti di Radetzky e agli scritti di Karl Schönhals che forniscono quindi il punto di vista austriaco.

Leggendo di volta il volta le note, si comprende il difficile e importante lavoro che l'autore ha realizzato nell'analisi bibliografica del suo oggetto di studio. I testi che analizza - e questo è un

\footnotetext{
${ }^{11}$ COMANDO DEL CORPO DI STATO MAGGIORE. UFFICIO STORICO, Relazioni e rapporti finali sulla campagna del 1849 nell'Alta Italia, Roma, Officina Poligrafica, 1911; Relazioni e rapporti finali sulla campagna del 1848 nella Alta Italia, vol. I, Roma, Lab. tip. del Comando del Corpo di Stato Maggiore, 1908; AA.VV., Relazioni e rapporti finali sulla campagna del 1848 nella Alta Italia, voll. II-III, Roma, Lab. tip. del Comando del Corpo di Stato maggiore, 1910; BRANCACCIO, Nicola, PAGANELLI, Carlo, RAGIONI, Rodolfo, Relazioni e rapporti finali sulla campagna del 1849 nella Alta Italia, Roma, Officina Poligrafica, 1911; RAGIONI, Rodolfo, GIACCHI, Nicolò, BRANCACCIO, Nicola, La campagna del 1849 nella Alta Italia, Roma, Ministero della Guerra, 1928.

${ }^{12}$ AGAZZI, Renato, La rivoluzione del 1848. La nascita della patria, cit., p. 11.
} 
altro dato significativo sulla difficoltà degli studi storico-militari del Risorgimento - sono prevalentemente della fine dell'Ottocento e dei primi del Novecento. Anche per quanto concerne la documentazione afferente ai molti rapporti e relazioni utilizzati e puntualmente indicati in nota, si tratta sempre e come indicato più sopra, di pubblicazioni a stampa raccolte in tomi editi ormai da oltre cento anni ${ }^{13}$. Parimenti, però, non compaiono particolari e interessanti indicazioni di ricerche archivistiche, e uno studio storico privo di approfondimenti delle fonti primarie ne risente sicuramente in termini di contributo innovativo alla ricerca storica. Alla disponibilità di una "biblioteca antiquaria" sarebbe stato interessante quindi affiancare una ricerca d'archivio quale unico mezzo per far emergere eventuali importanti scoperte o analizzare la documentazione dell'epoca alla luce di nuove tesi e interpretazioni.

Il volume presenta anche un inserto fotografico che fornisce immagini di tele che ritraggono singoli militari o immagini di battaglie. Per quanto le seconde possano apparire più sceniche, di particolare interesse risultano quelle relative ai singoli. Come per il testo, anche nell'inserto fotografico l'autore si sforza di dare lo stesso spazio sia ai piemontesi che agli austriaci e le immagini forniscono così indicazioni sulle divise e sugli armamenti in dotazione ai due schieramenti. Ci appare proficua la scelta di accompagnare il testo con queste immagini che spesso passano inosservate all'interno dei musei, mentre nel contesto dello studio proposto forniscono interessanti indicazioni storiche e collegamenti con lo scritto.

Un particolare che colpisce, infine, sono i nomi delle Brigate militari di cui ci dà conto Agazzi per entrambi gli schieramenti. Quei nomi accompagneranno lo svolgersi della storia nazionale fino ai giorni nostri.

Nel complesso, seppur con alcune riserve qui indicate, il volume si fa apprezzare oltre che per la precisa ricostruzione degli avvenimenti bellici - tratto peculiare di un buon testo di storia militare, dove spesso risulta importante descrivere le azioni anche fornendo un'indicazione oraria, come fatto da Renato Agazzi - per aver affiancato ad essi, con un giusto bilanciamento e permettendone una maggiore comprensione, anche alcune importanti indicazioni su ciò che circondò il puro evento militare. In questo senso le rivolte, il pensiero politico d'impronta liberale o repubblicana, l'adesione alla Lega Federativa di Stati Italiani o ad una Lega Italiana di cui ci rende conto l'autore, ben si coniugano con gli "ordini di battaglia", coi i "corpi d'armata o con la "fanteria di linea".

\footnotetext{
${ }^{13}$ Vedasi nota 11.
} 


\section{L'AUTORE}

Edoardo GRASSIA, diploma di laurea in Sociologia e laurea in Storia medievale, moderna e contemporanea presso l'Università La Sapienza di Roma, è dottorando di ricerca in Storia dell'Europa presso lo stesso ateneo. Si è occupato dell'Aviazione Legionaria da Bombardamento, da cui è nata la pubblicazione L'Aviazione Legionaria da bombardamento (Spagna 1936-1939). Iniziare da stanotte azione violenta su Barcellona (Roma, IBN Editore, 2009) e di Resistenza e guerra di Liberazione con lo studio Sabato Martelli Castaldi. Il generale partigiano (Milano, Mursia, 2016). È autore di alcuni articoli editi da «Diacronie. Studi di Storia Contemporanea». Membro della Società Italiana di Storia Militare (SISM), svolge regolarmente attività di studio e di ricerca in ambito storico-sociale e storico-militare.

URL: < http://www.studistorici.com/progett/autori/\#Grassia > 